\title{
Energetic Electrons Accelerated in Solar Particle Events
}

\author{
R. P. Lin \\ Space Sciences Laboratory, University of Berkeley, CA 94720
}

\begin{abstract}
New measurements of energetic solar electrons from the WIND spacecraft are reviewed, and the implications for particle acceleration mechanisms discussed. In non-relativistic electron $/{ }^{3} \mathrm{He}$-rich (so-called impulsive) events the electron energy spectrum is often found to extend below $-1 \mathrm{keV}$, indicating that acceleration occurs high in the corona. Comparison of the escaping electrons with the electrons producing the associated hard X-ray burst suggests that acceleration is occurring over a wide range of altitudes. For Large Solar Energetic Particle (LSEP, or so-called gradual) events, WIND observations show the low energy $\sim 1-10 \mathrm{keV}$ electron component is sometimes missing. In many LSEP events the electrons are released from the Sun up to $\sim 0.5$ hour later than the onset of the solar type III radio burst, and coronal transient waves are detected traveling across the Sun by the SOHO EIT instrument. Onset timing analyses show two types of LSEPs; in some events the first arriving $\sim 0.1-6 \mathrm{MeV}$ protons are released $\sim 0.5-2$ hours after the electrons and travel a path length of $\sim 1.2 \mathrm{AU}$ (essentially scatter-free), while in other events the protons are released at the same time as the electrons but appear to travel $-2 \mathrm{AU}$. If we assume the observed energetic particles are accelerated by a shock in front of an outward propagating fast CME, the electrons are accelerated earlier and lower in the corona $(\sim 0.5$ $R_{S u n}$ ) and the protons later and higher, $\sim 4 R_{S u n}$ for the first type of event, and from $\sim 4$ to $>\sim 10 R_{S u n}$, with the more energetic protons accelerated lower for the second type. In mid-2000 the High Energy Solar Spectroscopic Imager (HESSI) mission will be launched to provide detailed X-ray and gamma-ray imaging and spectroscopy observations to study particle acceleration and energy release processes at the Sun. Comparisons between HESSI and ACE/WIND should provide new insights into the origins of energetic solar particles.
\end{abstract}

\section{INTRODUCTION}

Observations of impulsive non-relativistic electron events at energies above $\sim 40 \mathrm{keV}[1,2]$ provided the first interplanetary evidence for electrons accelerated in solar particle events. No energetic solar ions were detected above background in these events, indicating the electron to proton $(e / p)$ ratio is very large. Soon thereafter energetic electrons up to relativistic energies were detected accompanying the "classical" Large Solar Energetic Particle (LSEP) events [3]. These events are dominated by energetic, $>10 \mathrm{MeV}$ protons, with small $e / p$ ratios at a given energy.

Later, high sensitivity measurements from the ISEE-3 spacecraft of electrons down to $2 \mathrm{keV}$ showed that impulsive acceleration of nonrelativistic electrons occurred, on average, several times a day or more during solar maximum making these the most common type of particle acceleration by the Sun [4]. Many of these non-relativistic electron events are unaccompanied by reported $\mathrm{H} \alpha$ flares, and many are observed only at energies below $\sim 15 \mathrm{keV}$. With more sensitive measurements accelerated ions were detected above background for some of these non-relativistic electron events. The associated ion emission is primarily at low energies $\left(\sim \mathrm{MeV} /\right.$ nucleon and below) and ${ }^{3} \mathrm{He}$-rich $[4,5]$; that is, the ions have ${ }^{3} \mathrm{He} /{ }^{4} \mathrm{He}$ ratios of order unity while the typical ratios for the solar atmosphere or solar wind are a few times $10^{-4}$. The soft $\mathrm{x}$-ray (SXR) bursts accompanying these events are typically impulsive, with duration $\sim<10$ minutes, so these nonrelativistic electron- ${ }^{3} \mathrm{He}$-rich events are sometimes called impulsive solar energetic particle events. As these electrons escape they produce solar and interplanetary type III radio bursts through beamplasma interactions (see [6] for review).

On the other hand, LSEP events usually occur after a large solar flare. Tens of LSEP events are detected per year near solar maximum. The observed ionization states of LSEP particles are typical of a $1-2 \times 10^{6} \mathrm{~K}$ plasma, suggesting that these come from the quiescent solar corona and/or solar wind. It is believed that shock waves driven by fast Coronal Mass Ejections (CMEs), propagating over a wide longitude range of the solar corona, are the

CP528, Acceleration and Transport of Energetic Particles Observed in the Heliosphere: ACE 2000 Symposium, edited by Richard A. Mewaldt, et al.

(C) 2000 American Institute of Physics 1-56396-951-3/00/\$17.00 

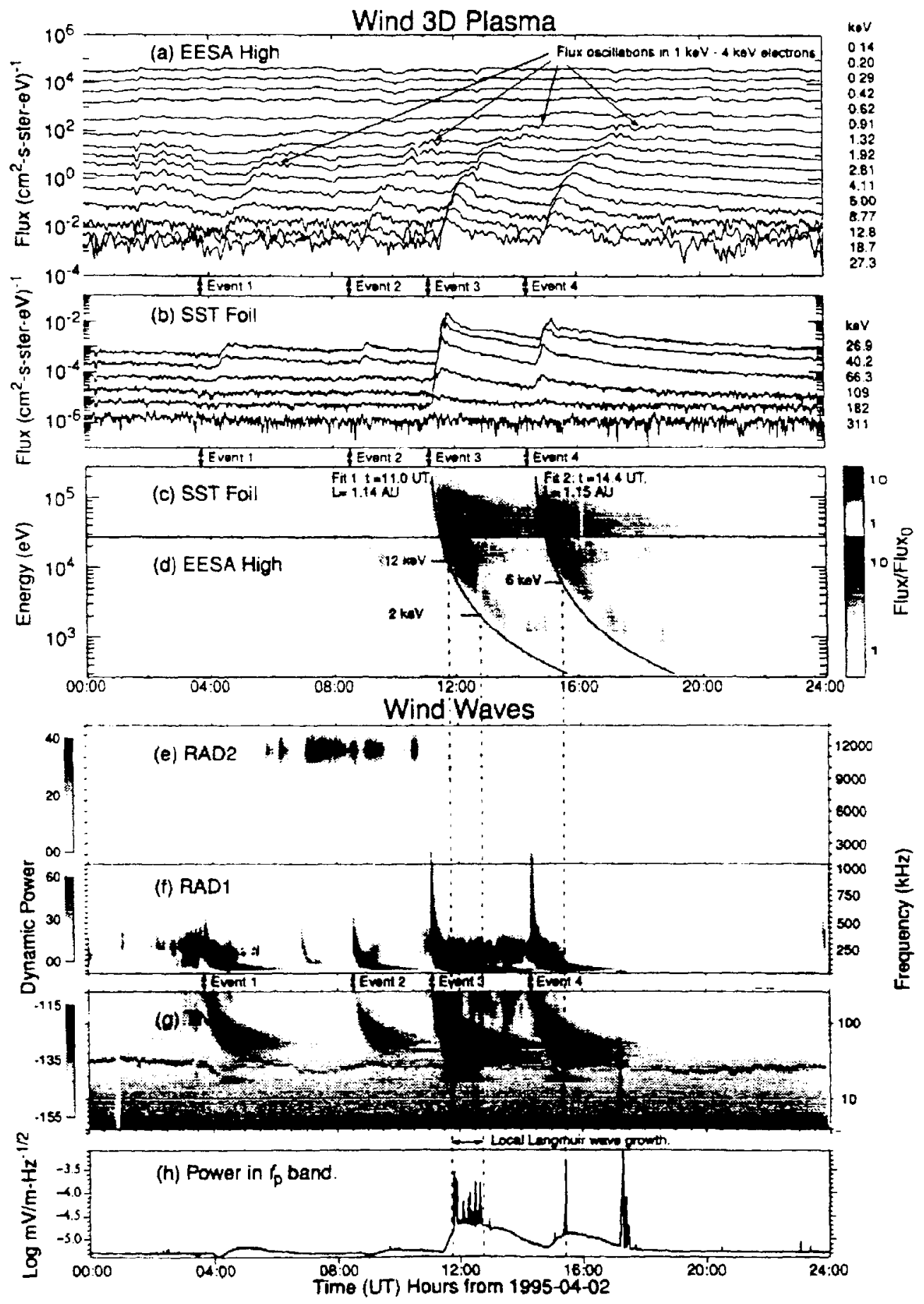

Figure 1. (a) The omnidirectional electron fluxes at 96 s resolution as measured by EESA-H on WIND. The center energies are listed on the right. (b) The omnidirectional electron fluxes as measured by the SST-Foil detector. (c) and (d) Spectrograms of the ratio $(\mathrm{F} / \mathrm{F} 0)$ of electron fluxes $(\mathrm{F})$ to a reference flux $(\mathrm{F} 0)$ prior to the first event. Superimposed on the plot are linear fits of the arrival time of the electrons versus their inverse velocity. The event start times are marked on the plot. (e), (f), and (g) Solar type III radio bursts as observed by the WIND WAVES instrument. The RAD2 panel displays the radio emissions from $1 \mathrm{MHz}$ to $14 \mathrm{MHz}$ (linear frequency axis). The TNR (Thermal Noise Receiver) panel displays the frequency range from $4 \mathrm{kHz}$ to 250 $\mathrm{kHz}$. (h) The electric field wave power in the frequency band (19 kHz to $41.5 \mathrm{kHz})$ encompassing the local Langmuir frequency From [7], (C) 1998. The American Astronomical Society. 
accelerating agent. LSEP events are also called "gradual" events since they are typically accompanied by flare soft X-ray (SXR) emission of relatively long duration, with e-folding decay times of $>\sim$ tens of minutes.

Since the launch of WIND and ACE, the new observations have changed our view of solar particle events. This paper reviews particle, $\mathrm{X}$-ray, radio observations relevant to electron acceleration in solar particle events.

\section{NONRELATIVISTIC ELECTRON- ${ }^{3} \mathrm{HE}-$ RICH EVENTS}

Figure $1 \mathrm{a}$ and $1 \mathrm{~b}$ show four solar impulsive electron events detected on April 2, 1995 [7] by the 3D Plasma and Energetic Particles Experiment [8] on the WIND spacecraft. This experiment was designed to bridge the gap between solar wind plasma and energetic particle measurements by providing high sensitivity, wide dynamic range, good energy and angular resolution, full 3-D coverage, and high time resolution over the energy range from a few $\mathrm{eV}$ to $>300 \mathrm{keV}$ for electrons and to $\rightarrow>6 \mathrm{MeV}$ for ions.

The impulsive events can be clearly identified through the velocity dispersion of the onsets, with the strongest event beginning at $\sim 1110$ UT at 182 $\mathrm{keV}$ and extending down in energy to $\sim 0.6 \mathrm{keV}$. In all of the events, velocity dispersion of the electrons (Figure 1c and 1d) is consistent with the expected Archimedean spiral length $(\sim 1.15 \mathrm{AU})$ for the measured solar wind velocity $\left(\sim 370 \mathrm{~km} / \mathrm{s}^{-1}\right)$.

All four of the solar impulsive electron events were associated with solar type III radio bursts (Figure 1e-1g) observed by the WIND WAVES instrument [9]. The narrow-banded emissions that persisted through out the day varying between 20 $\mathrm{kHz}$ and $40 \mathrm{kHz}$ (see Figure $1 \mathrm{~g}$ ) are locally generated Langmuir waves; the power in Langmuir emissions is shown in Figure 1h. It appears that the velocity dispersion produces electron distributions unstable to the generation of Langmuir waves. The Langmuir waves then produce radio emission through wave scattering. These observations confirm that the nonrelativistic electrons are the source of the emission, and therefore the radio bursts can be used to track the escaping electrons from the Sun into the interplanetary medium.

Figure 2 shows electron energy spectra for a typical impulsive event. The left-most spectrum shows the pre-event solar wind electron core and halo (dotted lines give Maxwellian fits), and shows that a highly non-thermal "superhalo" extends out to $\sim 100 \mathrm{keV}$ at this time. Due to velocity dispersion the peak in the event spectra progresses to lower energies with time; contributions from other smaller events are also evident, e.g., at 11:45-11:55 UT. The event-integrated spectrum displays a peak at $\sim<1$ $\mathrm{keV}$, with significant flux at $\sim 0.5 \mathrm{keV}$. Above the peak, the spectrum is similar to those reported previously above $-2 \mathrm{keV}$ [10], and can be fit to a power-law shape $\mathrm{d} J / \mathrm{d} E=A E^{-\delta}$, where $E$ is the electron energy in $\mathrm{keV}$ and $A$ and $\delta$ are constants. The best fit gives $\delta=3.0$ from $\sim 1 \mathrm{keV}$ to $40 \mathrm{keV}$, steepening to $\delta=4.4$ above $\sim 40 \mathrm{keV}$. Integrating over the energy spectrum and over the duration of the event, and assuming that the electrons are emitted into a $\sim 40^{\circ}$ cone of propagation in the interplanetary medium, we estimate a total energy of $\sim>3 \times 10^{26}$ ergs in escaping electrons.

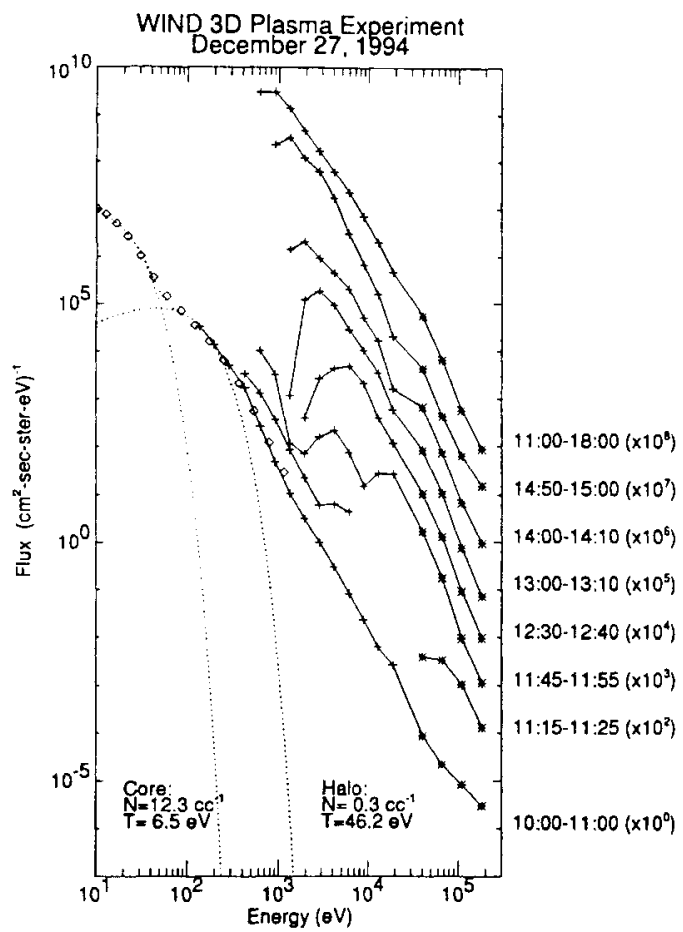

Figure 2. Omnidirectional electron spectra (with pre-event electron fluxes subtracted) are shown for various times during the 27 December 1994 event, and averaged over the entire event. The pre-event electron spectrum shows the solar wind electron core and halo components, as well as a 'super halo' extending to > 100 keV [11].

Because at coronal temperatures electrons are not gravitationally bound while protons are, an ambipolar electric field (the Pannekoek-Rosseland field $[12,13])$ is set up with a total potential drop of about $1 \mathrm{kV}$ from the base of the corona to $1 \mathrm{AU}$. 
This potential varies inversely with distance from the center of the Sun. It accelerates protons outward and decelerates electrons. Thus, the peak in the spectrum of the electrons just as they escape the corona could be up to $\sim 1 \mathrm{keV}$ more than measured at $1 \mathrm{AU}$, e.g., ranging from $\sim 1$ up to $\sim 2 \mathrm{keV}$ for the event of Figure 2, depending on the height of the acceleration.

The fact that the event spectrum extends down to such low energies indicates that at least some of the electron acceleration must occur high in the corona, since the range of $\sim \mathrm{keV}$ energy electrons in ionized hydrogen, due to Coulomb collisions, is short compared to the column depth through the corona. Assuming that the initial accelerated electron spectrum is represented by a power-law with the same exponent as seen at energies above the peak, the maximum overlying column density can be calculated [14]. For a peak at $\sim 1.5 \mathrm{keV}$, the column density must be less than $\sim 9 \times 10^{17} \mathrm{~cm}^{-2}$. This value implies that the lowest energy electrons must have been accelerated at altitudes of $\sim 1 R_{\text {Sun }}$, for the typical active coronal density models [15], or $\sim 0.2$ $R_{\text {Sun. }}$ for the quiet equatorial corona at sunspot minimum [16].

A ${ }^{3} \mathrm{He}$ enhancement was detected in solar energetic ions during this electron event (J. Mazur, private communication, 1995). If the ${ }^{3} \mathrm{He}$ originated at the same altitude in the corona as these low energy electrons, the magnetic field would likely be much too weak for acceleration by electromagnetic hydrogen cyclotron waves [17].

\section{ELECTRON - HARD X-RAY COMPARISON}

The escaping electron population can be compared to those electrons at the Sun, inferred from the bremsstrahlung $\mathrm{X}$-ray emission they produced through collisions. These comparisons show the number of escaping electrons is generally $10^{-2}$ to $10^{-3}$ the number of electrons at the Sun [4]. The typical numbers of electrons in an interplanetary event, however, would produce too few X-rays at the Sun for the present hard X-ray instrumentation to detect. On the other hand, even the largest electron events observed at $1 \mathrm{AU}$ have far too few electrons to produce the observed hard X-ray emission in a large flare.

Direct comparison of the X-ray producing electrons and escaping electrons from a solar flare on 8 November, 1978 [18], showed that the energy spectrum of the escaping electrons observed from 2 to $100 \mathrm{keV}$ differs significantly from the spectra of the X-ray producing electrons (Figure 3 ) and of the accelerated electrons (computed assuming that energy loss by Coulomb collisions is the dominant electron loss process). Even taking into account the material traversed by the escaping electrons, we find that they could not have come directly from the acceleration region. The accelerated electron spectrum at $>25 \mathrm{keV}$ energies, where energy losses are negligible, is much steeper than the escaping electron spectrum. If we match the accelerated and escaping spectra at $25 \mathrm{keV}$, a discrepancy of several orders of magnitude results at low energies. If the amount of material traversed is increased to reduce the low energy flux, the escaping spectrum would bend over in the energy range $2-10 \mathrm{keV}$.

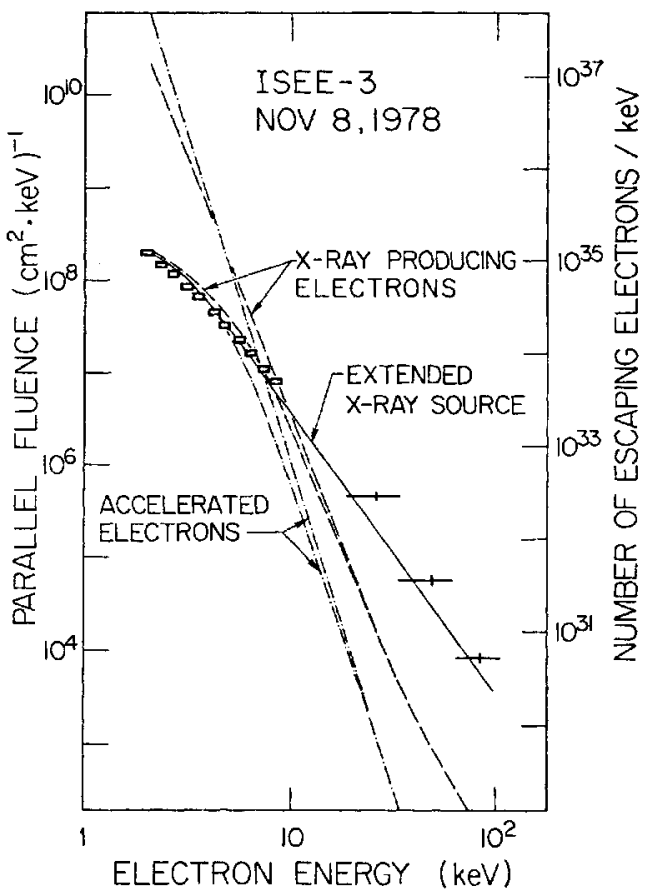

Figure 3. The spectra of the X-ray producing electrons (dashed lines) and the accelerated electrons (dash-dot lines) are shown here for comparison with the escaping electron spectrum (boxes and crosses) observed at 1 AU. The lower curved spectra of each pair of lines show how energy loss from the passage of the escaping electrons through the overlying solar atmosphere might modify these spectra. The solid curve is computed for electrons escaping from and $\mathrm{X}$-ray source which extends from the chromosphere to high in the corona ${ }_{4}$ From [18], with kind permission from Kluwer Academic Publishers.

The observations suggest a model where the escaping electrons come from an extended X-ray producing region which ranges from the chromosphere to high in the corona. In this model the low energy escaping electrons $(2-10 \mathrm{keV})$ come 
from the higher part of the extended X-ray source where the overlying column density is low, while the high energy electrons $(20-100 \mathrm{keV})$ come from the entire X-ray source.

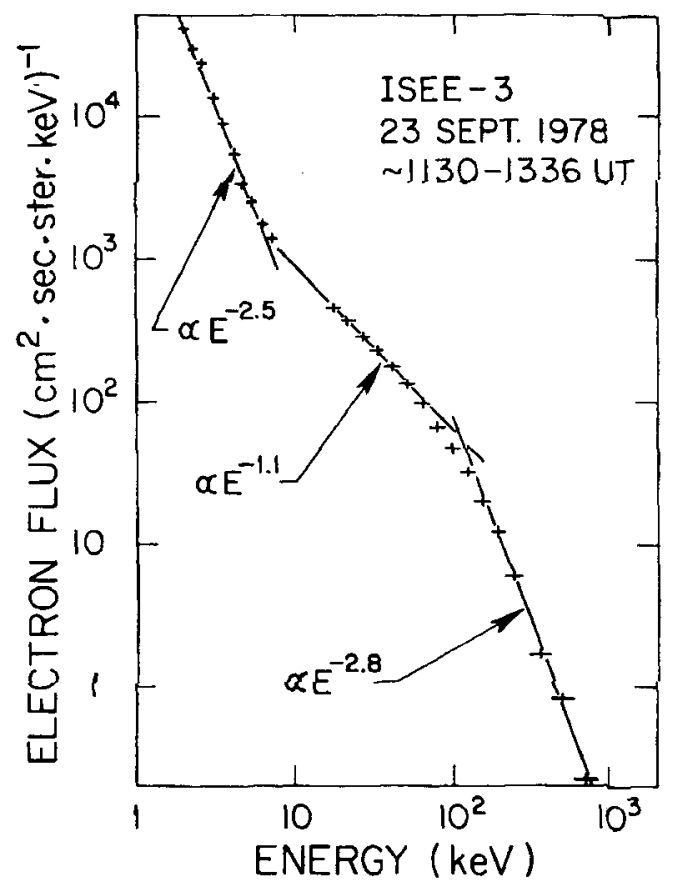

Figure 4. The energy spectrum for the large solar flare event of 23 September, 1978 as observed by ISEE-3.

\section{LSEP ELECTRON ACCELERATION}

The $20 \mathrm{keV}-20 \mathrm{MeV}$ energy spectra for LSEP electrons [19] typically show a double power law with a smooth transition around $100-200 \mathrm{keV}$ and power law exponents of 0.6-2.0 below and 2.4-4.3 above. The more intense the event, the harder the spectrum. ISEE-3 observations at energies down to $-2 \mathrm{keV}$ showed that most LSEP events near solar maximum had a low-energy component with a steep power law spectrum (Fig. 4) extending from $\sim 10 \mathrm{keV}$ down to $<\sim 2 \mathrm{keV}$ [4], similar to the spectra of nonrelativistic electron events. This was taken as evidence that in LSEP events the acceleration accurred high in the corona, as would be expected for CMEs. The three events found without this low energy component were those with impulsive SXR bursts $[20]$.

Figure 5 shows the 22 April 1995 event observed by WIND. The presence of significant fluxes of $>10$ $\mathrm{MeV}$ protons and the gradual SXR burst confirm this is an LSEP event. Electrons are detected down to $\sim 8$ $\mathrm{keV}$ in this event (Fig. 5) with no increase below $\sim 6$
$\mathrm{keV}$. The electron spectrum for the event (Figure 6) shows a peak at $\sim 12 \mathrm{keV}$, and a rapid falloff below $\sim 8 \mathrm{keV}$. Above $\sim 12 \mathrm{keV}$ the spectrum fits a double power law with a break around $50 \mathrm{keV}$, and power law exponents of $\delta=1.1$ below and $\delta=3.1$ above the break.

If the peak is due to the electrons traversing overlying material, the source region must be at a column depth of $\sim 1 \times 10^{19} \mathrm{~cm}^{-2}$. For quiet coronal density models the electrons would have been accelerated in the chromosphere, while for active corona models the height would be $\sim 0.15 R_{\text {Sun }}$. Thus, this event is inconsistent with the simple picture for LSEP events of particle acceleration by CME shocks high ( $>\sim$ few $R_{\text {Sun }}$ ) in the corona. In surveying the WIND data, a number of such LSEP events, with no low energy $(<\sim 10 \mathrm{keV})$ electron emission, have been found [21].

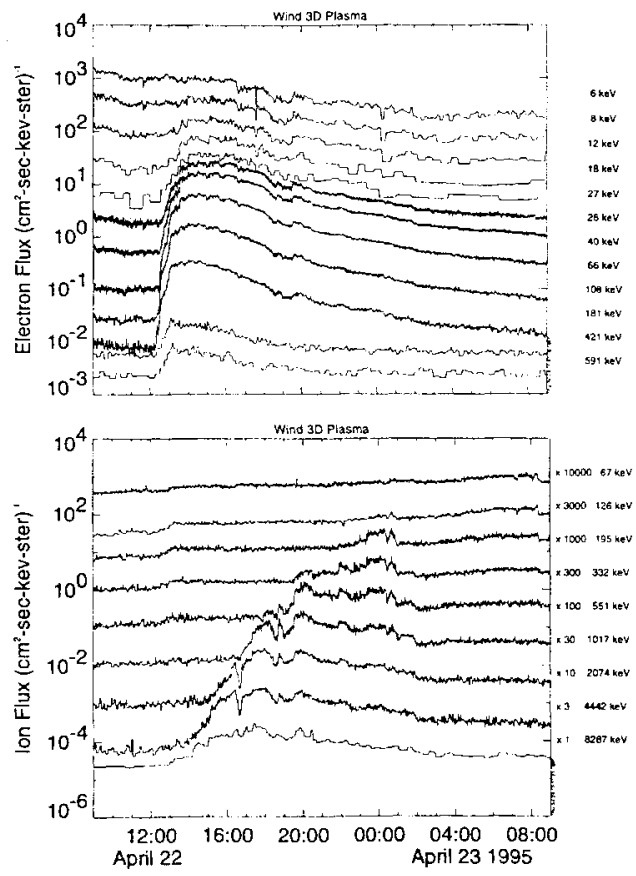

Figure 5. Energetic electron (upper panel) and ion (lower panel) fluxes for 22-23 April 1995. The energies (and flux factors) are indicated on the right. The gradual (LSEP) event is evident at energies above $\sim 12 \mathrm{keV}$ in electrons.

\section{ONSET TIMING STUDIES}

Krucker et al. [21] surveyed the solar electron events observed by Wind. Timing analysis of the velocity dispersion of the onsets reveals two different kinds of electron events: (1) events released from the Sun at the onset of a radio type III burst, which 
suggest that these electrons are part of the population producing the type III radio emission; and (2) events in which the electrons are released up to half an hour later than the onset of the type III burst. The latter events are proton rich, i.e. LSEP events.

For about $3 / 4$ of the delayed LSEP events, largescale coronal transient waves, also called EIT waves or coronal Moreton waves, are observed by the Extreme Ultraviolet Imaging Telescope (EIT) on board SOHO. These waves originate at the flare site and often traversed a substantial fraction of the solar disk to reach the footpoint of the spiral interplanetary field line connected to the Wind spacecraft. It's not clear whether these waves are the chromospheric/lower coronal signatures of $\mathrm{CME}$ shocks or a separate flare driven shock.

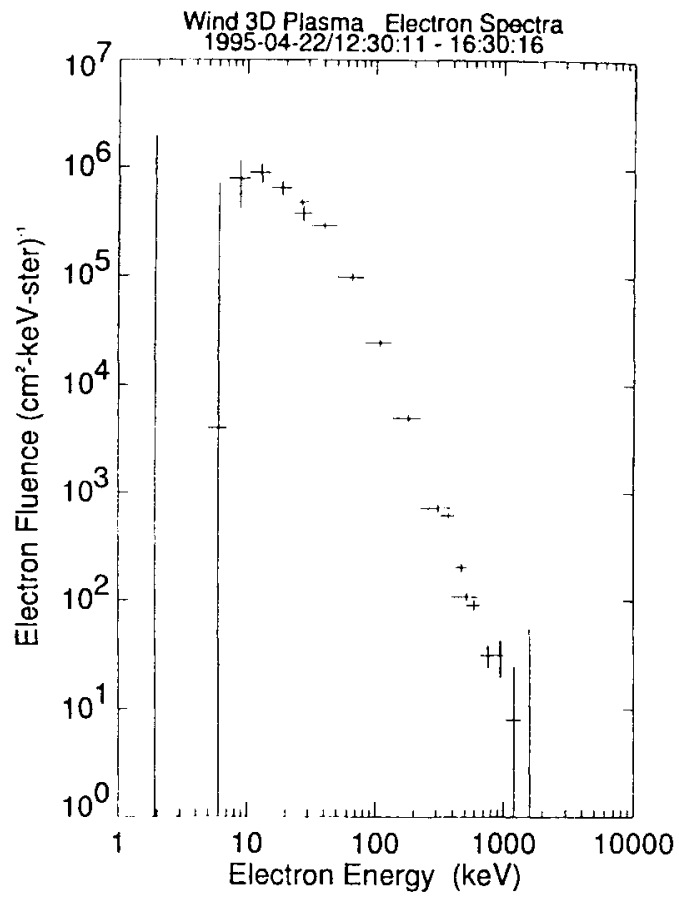

Figure 6. Omnidirectional electron energy spectrum (with pre-event background subtracted) averaged over the first four hours of the event.

Recently, Krucker and Lin [22] analyzed the velocity dispersion of $\sim 30 \mathrm{keV}-6 \mathrm{MeV}$ proton and $\sim 1-300 \mathrm{keV}$ electrons in LSEP type events. Two classes of events were found. For one class (70\% of the events), the first arriving protons and electrons travel essentially scatterfree, with derived path lengths between 1.1 and $1.3 \mathrm{AU}$, but the protons are released $\sim 0.5$ to 2 hours later. For events of the second class the protons show significantly larger path lengths, around $2 \mathrm{AU}$, than the electrons (still $\sim 1.2 \mathrm{AU}$ ) but the protons and electrons are to be released simultaneously, within an uncertainty of 20 minutes.

For both classes of events the ions in the onset phase are observed to be streaming outward, aligned within $\sim 45^{\circ}$ pitch angle of the magnetic field, with no obvious difference between classes. Thus, it is unlikely that pitch angle scattering can explain the difference in path lengths. The alternative explanation is that the acceleration/ injection of protons varies monotonically with energy, with the highest energy protons accelerated/ injected earliest.

\section{SUMMARY}

It appears that non-relativistic (impulsive SXR) electron events are generally the result of an acceleration that occurs high in the corona. The electrons then produce type III radio emission as they escape from the Sun. Whether this acceleration is part of the acceleration of the electrons which produce the hard X-ray burst, or a simultaneous but separate phenomenon, is not clear. How these are related to the acceleration of ${ }^{3} \mathrm{He}$ is also unclear.

The LSEP electron acceleration process sometimes produces only higher energy electrons, from $\sim 8-10 \mathrm{keV}$ to relativistic energies. The simplest interpretation of the electrons from $<\sim 1 \mathrm{keV}$ to $\sim 10$ $20 \mathrm{keV}$ sometimes seen in LSEP events (and sometimes not) is that they are due to a mixed event, where the non-relativistic (impulsive SXR) electron acceleration occurs in the LSEP event.

The LSEP electron acceleration appears related to the EIT wave which may or may not be related to the CME shock. If the CME shock accelerates LSEP electrons and ions, the ions appear to be accelerated later, at a higher altitude than the electrons. For one class of events, ions of all energies are accelerated at $\sim 4 R_{\text {Sun }}$ altitude. For the second class the ions are accelerated at altitudes from $\sim 4$ to $>\sim 10 R_{\text {Sun }}$, with the lower energy ions accelerated higher. The reasons for the two classes are not presently understood.

Clearly, spatially resolved observations of X-ray and $\gamma$-ray continuum and $\gamma$-ray line signatures of energetic electrons and ions, respectively, at the Sun, will help to identify the acceleration mechanisms. The High Energy Solar Spectroscopic Imager (HESSI) planned for launch in July 2000 [21], will provide such observations for comparison with solar 
particle measurements from ACE, WIND, and other spacecraft.

\section{ACKNOWLEDGMENTS}

This research was supported in part by NASA grants NAG5-6928 and NAG5-2815.

\section{REFERENCES}

1. Van Allen, J. A., and S. M. Krimigis, J. Geophys. Res. 70, 5737- (1965).

2. Anderson, K.A., and R.P. Lin, Phys. Rev. Lett. 16, 1121-1124 (1966).

3. Cline, T. L., and F. B. McDonald, Solar Phys. 5, $507-$ 530 (1968).

4. Lin, R. P., Solar Phys. 100, 537-561 (1985).

5. Reames, D.V., and T.T. von Rosenvinge, and R.P. Lin, Astrophys. J. 292, 716-724 (1985).

6. Lin, R.P., 'Electron beams and Langmuir turbulence in solar Type III radio bursts observed in the interplanetary medium', in Basic Plasma Processes on the Sun, edited by E. Priest and V. Krishan, International Astronomical Union, The Netherlands, 1990, pp.467-481.

7. Ergun, R.E., D. Larson, R.P. Lin, J.P. McFadden, C.W. Carlson, K.A. Anderson, L. Muschietti, M. McCarthy, G. Parks, H. Reme, J. M. Bosqued, C. d'Uston, T.R. Sanderson, K.P. Wenzel, M. Kaiser, R.P. Lepping, S.D. Bale, P. Kellogg, and J.-L. Bougeret, Astrophys. J. 503, 435-445 (1998).

8. Lin, R. P., K. A. Anderson, S. Ashford, C. Carlson, D. Curtis, R. Ergun, D. Larson, J. McFadden, M. McCarthy, G. K. Parks, H. Rème, J.M. Bosqued, J. Coutelier, F. Cotin, C. d'Uston, K.-P. Wenzel, T.R. Sanderson, J. Henrion, and J.C. Ronnet, Space Sci. Rev. 71, 125-153 (1995).

9. Bougeret, J.-L., M.L. Kaiser, P.J. Kellogg, R. Manning, K. Goetz, S.J. Monson, N. Monge, L. Friel, C.A. Meetre, C. Perche, L. Sitruk, and S. Hoang, Space Sci. Rev. 71, 231-263 (1995).

10. Potter, D. W., R. P. Lin and K. A. Anderson, Astrophys. J. 236, L97-L100 (1980).

11. Lin, R. P., D. Larson, J. McFadden, C.W. Carlson, R.E. Ergun, S. Ashford, K.A. Anderson, M. McCarthy, G.K. Parks, H. Rème, J.M. Bosqued, C. d'Uston, T.R.
Sanderson, and K.-P. Wenzel, Geophys. Res. Lett. 23, 1211-1214, 1996.

12. Pannekoek, A., 'Ionization in stellar atmospheres', Bull. Astron. Inst. Neth., 1, 107-(1922).

13. Rosseland, S., Monthly Notices Royal Astron. Soc. 84, 720-, (1924).

14. Lin, R.P., Space Sci. Rev. 16, 189-256 (1974).

15. Dulk, G.A., and D.J. McLean, Solar Physics 57, 279 295 (1978).

16. Saito, K., A. I. Poland, and R. H. Munro, Solar Phys. 55, 121-134 (1977).

17. Temerin, M., and I. Roth, Astrophys. J. 391, L105L108 (1992).

18. Pan, L., R.P. Lin, and S.R. Kane, Solar Phys. 91, 345357 (1983).

19. Lin, R. P., R. A. Mewaldt, and M. A. I. Van Hollebeke, Astrophys. J. 253, 949-962 (1982).

20. Lin, R. P., "Electron energy spectra from $2 \mathrm{keV}$ to 1 $\mathrm{MeV}$ for large solar flare events," in 21 st Internat'l. Cosmic Ray Conf. Papers, edited by R. J. Protheroe, International Cosmic Ray Conference Committee, 1990, pp. 88-91.

21. Krucker, S., D. E. Larson, R. P. Lin, and B. J. Thompson,. Astrophys. J. 519, 864-875 (1999).

22. Krucker, S., and R. P. Lin, these proceedings.

23. Lin, R. P., these proceedings. 